\title{
Left Ventricular Noncompaction with Bicuspid Aortic Valve: A Case Report with a Comprehensive Cardiac MRI Approach
}

\author{
Woo Jin Yang ${ }^{1,2}$, Jung Im Kim ${ }^{3,{ }^{*}}$, Eun-Sun Jin ${ }^{4}$, Han Na Lee ${ }^{1}$ and So Youn Shin ${ }^{5}$ \\ ${ }^{1}$ Department of Radiology, Kyung Hee University Hospital at Gangdong, Seoul, Korea \\ ${ }^{2}$ Department of Radiology, Asan Medical Center, Seoul, Korea \\ ${ }^{3}$ Department of Radiology, Kyung Hee University Hospital at Gangdong, College of Medicine, Kyung Hee University, Seoul, Korea \\ ${ }^{4}$ Cardiovascular Center, Kyung Hee University Hospital at Gangdong, Kyung Hee University, Seoul, Korea \\ ${ }^{5}$ Department of Radiology, Kyung Hee University Hospital, College of Medicine, Kyung Hee University, Seoul, Korea \\ "Corresponding author: Department of Radiology, Kyung Hee University Hospital at Gangdong, College of Medicine, Kyung Hee University, 892 Dongnam-Ro. Gangdong-Gu, \\ Seoul, 05278, Korea. Tel: +82-24406933, Fax: +82-24406932, Email: mine147@gmail.com
}

Received 2018 October 25; Revised 2019 April 23; Accepted 2019 May 04.

\begin{abstract}
Noncompaction of ventricular myocardium, once thought to represent a rare cardiomyopathy, is being diagnosed with increased frequency. It is often accompanied by various congenital heart diseases. Coexisting valvular anomalies are an emerging issue that requires further investigation in patients with noncompaction cardiomyopathy, because they can increase the potential for adverse clinical events. This article describes the case of a 45-year-old male diagnosed with left ventricular noncompaction (LVNC) with a bicuspid aortic valve. Progressive deterioration of cardiac function in this patient could be explained by myocardial fibrosis and accompanying valvular anomalies on cardiac magnetic resonance imaging (MRI). The clinical implications of cardiac MRI in noncompaction cardiomyopathy are discussed based on its ability to depict both morphological and functional features. Considering the potential for coexisting congenital heart disease to declines in cardiac function, patients with LVNC should be comprehensively evaluated for concurrent cardiac anomalies and myocardial fibrosis.
\end{abstract}

Keywords: Noncompaction, Bicuspid Aortic Valve, Magnetic Resonance Imaging, Echocardiography, Transesophageal

\section{Introduction}

Noncompaction of ventricular myocardium is the description of a myocardial phenotype with a severely thickened myocardium presenting with a prominent noncompacted layer (excessive trabeculations, and deep intertrabecular recesses), which is at least twice as thick as the compacted layer (1). It is being detected with increased frequency given the rising awareness of the characteristic features of echocardiography and cardiac magnetic resonance imaging (MRI) (2). Although the pathogenesis of noncompaction of myocardium remains unclear, one of the most widely accepted hypotheses is that noncompaction results from arrest in the normal compaction process of ventricular endomyocardial development. The left ventricle is commonly involved, while less than $50 \%$ of cases exhibit biventricular involvement (3).

Myocardial noncompaction can be isolated or associated with congenital heart anomalies such as ventricular septal defect (VSD), atrial septal defect (ASD), pulmonic stenosis (PS), double orifice mitral valve (DOMV), mitral stenosis (MS), bicuspid aortic valve (BAV), and Ebstein anomaly (4). This report describes a patient with left ventricular noncompaction (LVNC) and BAV in whom cardiac MRI comprehensively depicted myocardial fibrosis, asymmetrical septal hypertrophy and coexisting valvular anomaly.

\section{Case Presentation}

A 45-year-old man presented to the emergency room (ER) with altered mentality and ventricular fibrillation. The patient had a similar episode of syncope 7 years prior, but refused further evaluation at that time. The patient did not have a family history of heart disease. Chest x-ray revealed severe cardiomegaly and suspected pulmonary edema.

For exclusion of coronary heart disease, emergent cardiac catheterization was performed and there was neither abnormal stenosis nor thrombus of the coronary arteries. Two-dimensional and transesophageal echocardiography (TEE) demonstrated asymmetrical septal hypertrophy, dilated left heart chambers and prominent trabeculations and deep recesses in the apico-mid lateral wall of the left ventricle, suggesting noncompaction cardiomyopathy (Figure 1A). 

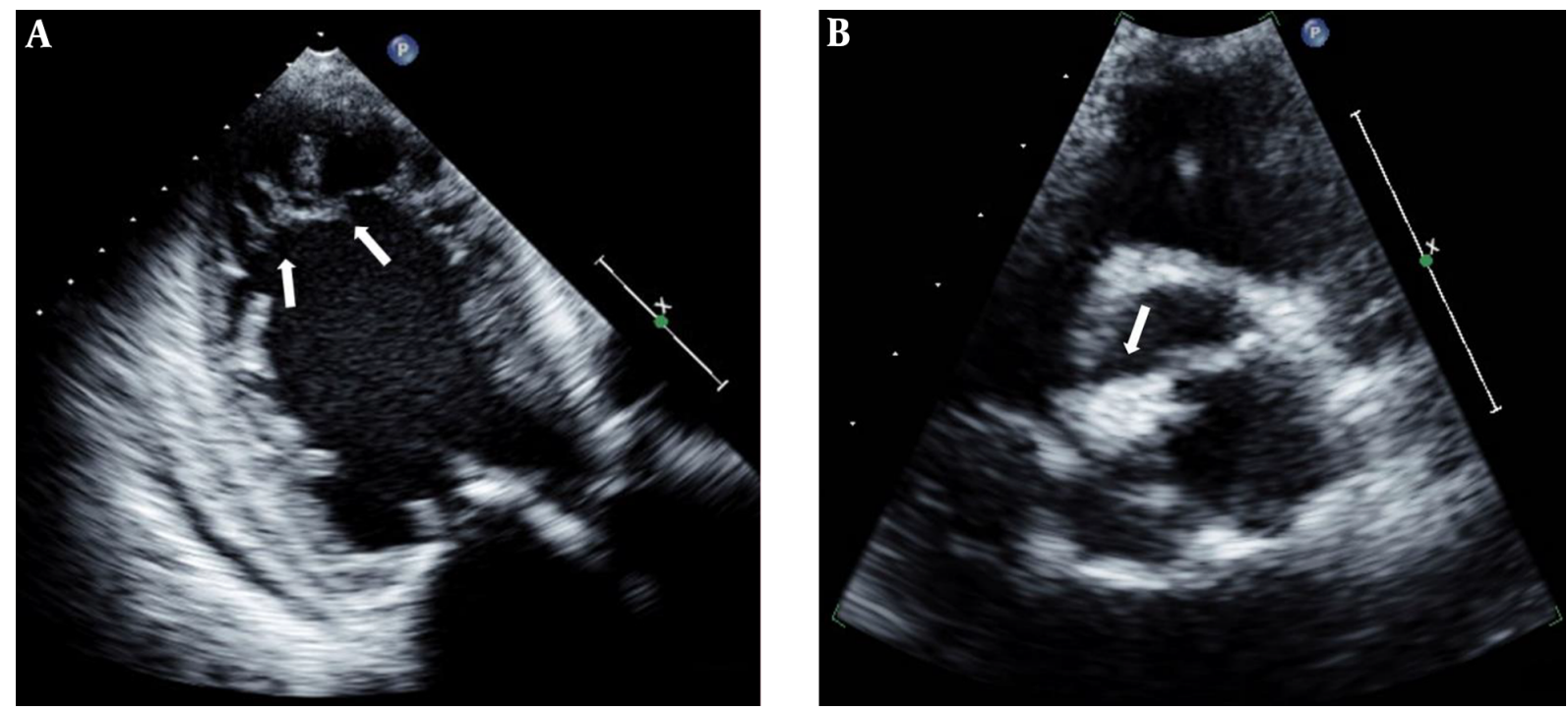

Figure 1. Transesophageal apical four-chamber view revealed a dilated left ventricle(LV) and noncompacted myocardium (arrows) in the midventricular and apical segments of the LV (A). On the midesophageal diastolic short axis view, atypically configured aortic leaflets with commisural fusion (arrow) of the right coronary and noncoronary leaflets are observed (B).

BAV with moderate aortic regurgitation (AR) (Figure 1B) and thickened mitral valves with mild mitral regurgitation (MR) were also identified, but systolic function was preserved (ejection fraction [EF] 67\%). During 24-h electrocardiogram (ECG) monitoring, non-sustained ventricular tachycardia was observed.

Delayed gadolinium-enhanced cardiac MRI revealed asymmetric hypertrophy of the interventricular septum and noncompacted myocardium in the lateral and apical walls of the left ventricle (LV), in which transmural myocardial and trabecular enhancement was noted presumably due to myocardial fibrosis and scarring (Figure 2A and B). The maximum end-diastolic ratio of non-compacted to compacted myocardial wall thickness was 3.1:1. On shortaxis cine images, focal fusion of the right coronary cusp and noncoronary cusp suggesting BAV was identified (Figure $2 \mathrm{C}$ ). In addition, mitral valves were thickened, accompanied by calcification. After intensive treatment for decompensated heart failure, a cardioverter-defibrillator pacemaker was implanted. While he was followed by echocardiography for 3.5 years, dilation of the left heart chamber and LV systolic dysfunction progressed (ejection fraction [EF]: 35\%), AR and MR were aggravated, and pulmonary hypertension developed.

\section{Discussion}

LVNC has been classified as a primary genetic cardiomyopathy. It occurs more frequently in males and its prevalence ranges from $0.06 \%-0.24 \%$; nearly half of the cases are familial (5). Recently, clinicians and researchers have debated whether LVNC is a physiologic or a pathologic phenotype of the myocardium. LVNC can be a myocardial disorder (genetic cases), as in patients with hypertrophic cardiomyopathy or dilated cardiomyopathy. It can be an epiphenomena of a pathologic pressure/volume load of the ventricle, or it can be a physiologic response to loading conditions $(1,6)$.

Although many patients are asymptomatic with preserved LV systolic function, others develop heart failure, thromboembolic events, and malignant arrhythmias. Heart failure symptoms varying from mild to severe have been observed in approximately half of patients (7). Systolic dysfunction and arrhythmias may be a consequence of dysfunction at the microcirculation level and subepicardial hypoperfusion (4).

Echocardiography is the main diagnostic tool for the diagnosis of noncompaction. Especially, TEE might have a complementary role in the detection of LVNC, particularly in adult patients, in whom this unusual condition may be misdiagnosed. Cardiac MRI is becoming increasingly important in confirming the diagnosis and evaluating concomitant complications and myocardial scarring. Advantages of cardiac MRI compared with echocardiography for diagnosing LVNC include the high spatial resolution; better delineation between the noncompacted and compacted layer; the ability to visualize the apex, false tendons, prominent papillary muscles, and aberrant bands 

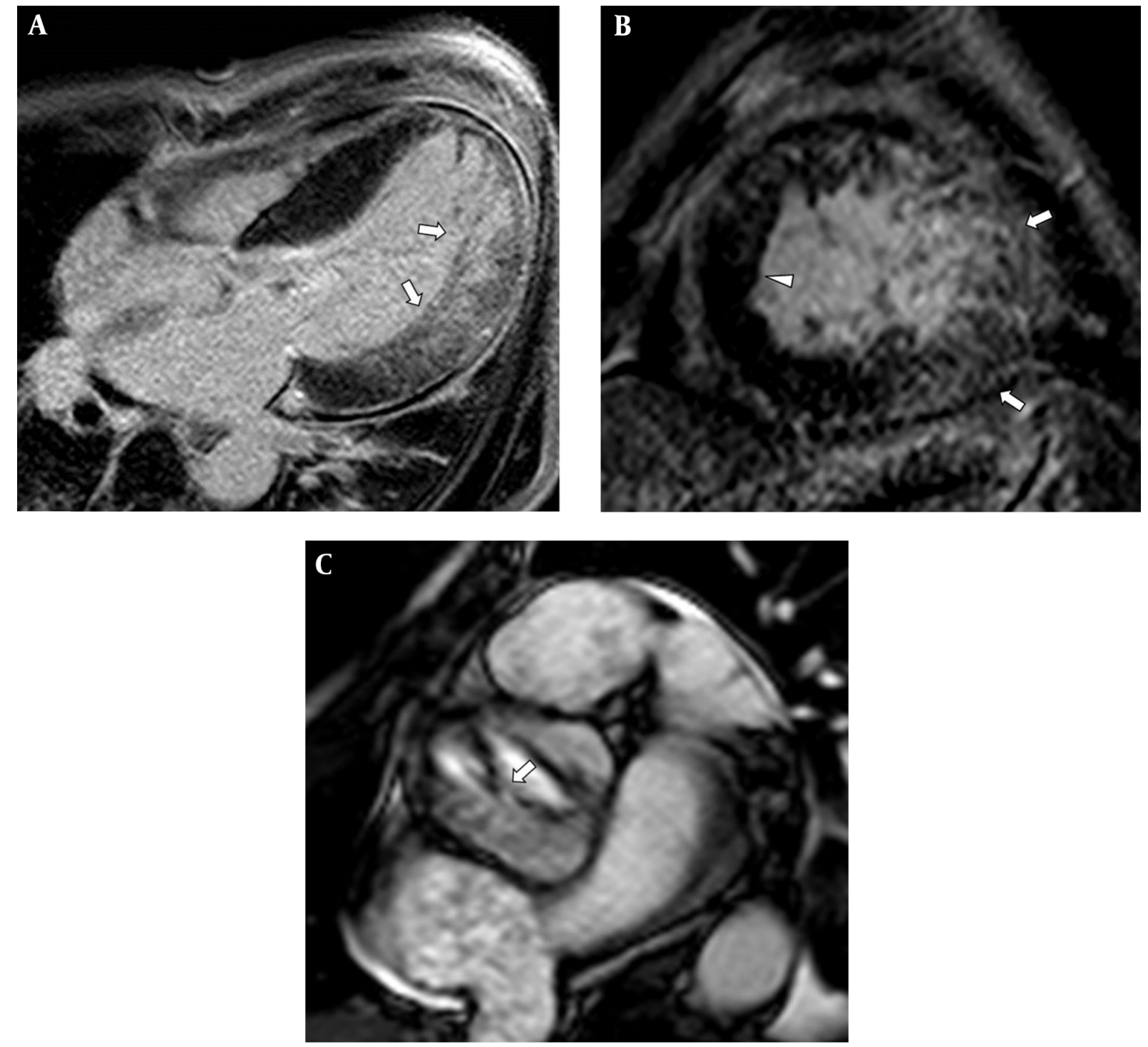

Figure 2. Four-chamber (A) and short-axis (B) delayed-enhancement MRI revealed hypertrabeculated noncompacted myocardium in the midventricular and apical left ventricle (LV), which demonstrated patchy trabecular and myocardial delayed enhancement (arrows). An asymmetrically hypertrophied LV septum was also noted (arrowhead). Short-axis cine-MRI (C) demonstrated a thickened bicuspid aortic valve with right-noncoronary leaflet fusion (arrow).

(8). Furthermore, the assessment of myocardial fibrosis on delayed gadolinium enhancement on cardiac MRI can provide valuable prognostic information. Nucifora et al. revealed that myocardial fibrosis on delayed-enhancement MRI is related to clinical disease severity and LV systolic dysfunction in isolated LVNC (9). An abnormal contractile mechanism and inadequate microvasculature in the setting of increased myocardial mass have been postulated as potential causes of fibrosis given the usual finding of normal epicardial coronary arteries (10). In this case, extensive delayed myocardial and trabecular enhancement of the non-compacted myocardium might be closely related with gradual deterioration of cardiac function on followup echocardiography.

Coexisting valvular anomalies are an emerging association with LNVC that requires further investigation, because it can attribute to expedite the potential for adverse clinical events. BAV is a common congenital cardiac malformation in adults, and it occurs in isolation, or in association with other congenital heart diseases. In Agarwal et al.'s study, an $11 \%$ incidence of LVNC was noted within the $B A V$ population, which was significantly higher than in the 
general population (11). Several cases illustrate an unusual association of noncompaction cardiomyopathy with morphologic and functional abnormalities of the mitral valve in the form of thickened leaflets with restricted movement (12). In these reports, the authors noted that atrioventricular valve abnormalities seemed to be secondary to valvular dysplasia or chordal enlongation or rupture, which could be related to endocardial fibroelastosis or papillary muscular involvement (12).

Prognosis is variable in patients with LVNC. Although some patients remain asymptomatic, rapid deterioration of cardiac function can result in early death (4). Considering the potential for coexisting congenital heart disease to contribute to declines in cardiac function, patients with LNVC should be comprehensively evaluated for concurrent cardiac anomalies and myocardial fibrosis using echocardiography and cardiac MRI.

\section{Footnotes}

Authors' Contributions: Jung Im Kim made substantial contributions to conception and design. Woo Jin Yang was a major contributor in writing the manuscript. Han Na Lee and So Youn Shin analyzed and interpreted the patient data regarding follow up of simple radiograph images and CT images. Eun-Sun Jin supervised the clinical course of the patient and selected appropriate echographic images. All authors read and approved the final manuscript.

Conflict of Interests: The authors declare that they have no conflict of interest.

Ethical Approval: The present case study was approved by the Kyung Hee University Hospital at Gangdong Institutional Review Board (Seoul, Korea).

Financial Disclosure: None of the authors have any financial interests relevant to the manuscript.

Funding/Support: None declared.

Patient Consent: The patient involved in this case provided written informed consent.

\section{References}

1. Oechslin E, Jenni R. Left ventricular noncompaction: From physiologic remodeling to noncompaction cardiomyopathy. J Am Coll Cardiol. 2018;71(7):723-6. doi: 10.1016/j.jacc.2017.12.031. [PubMed: 29447732].

2. Dursun M, Agayev A, Nisli K, Ertugrul T, Onur I, Oflaz H, et al. MR imaging features of ventricular noncompaction: Emphasis on distribution and pattern of fibrosis. Eur J Radiol. 2010;74(1):147-51. doi: 10.1016/j.ejrad.2009.01.015. [PubMed: 19328640].

3. Corrado G, Santarone M, Miglierina E, Beretta S, Frattini T, Tadeo G, et al. Isolated noncompaction of the ventricular myocardium. A study in an adult male and literature review. Ital Heart J. 2000;1(5):372-5. [PubMed: 10832816].

4. Dogan A, Aksoy H. Coexistence of congenital heart anomalies with noncompaction of the ventricular myocardium. Two case reports. Herz. 2012;37(6):699-701. doi: 10.1007/s00059-012-3582-4. [PubMed: 22301732].

5. Klaassen S, Probst S, Oechslin E, Gerull B, Krings G, Schuler P, et al. Mutations in sarcomere protein genes in left ventricular noncompaction. Circulation. 2008;117(22):2893-901. doi: 10.1161/CIRCULATIONAHA.107.746164. [PubMed: 18506004].

6. Arbustini E, Favalli V, Narula N, Serio A, Grasso M. Left ventricular noncompaction: A distinct genetic cardiomyopathy? J Am Coll Cardiol. 2016;68(9):949-66. doi: 10.1016/j.jacc.2016.05.096. [PubMed: 27561770].

7. O'Donnell DH, Abbara S, Chaithiraphan V, Yared K, Killeen RP, Martos $\mathrm{R}$, et al. Cardiac MR imaging of nonischemic cardiomyopathies: Imaging protocols and spectra of appearances. Radiology. 2012;262(2):40322. doi: 10.1148/radiol.11100284. [PubMed: 22282181].

8. Finsterer J, Stollberger C, Towbin JA. Left ventricular noncompaction cardiomyopathy: Cardiac, neuromuscular, and genetic factors. Nat Rev Cardiol. 2017;14(4):224-37. doi: 10.1038/nrcardio.2016.207. [PubMed: 28079110].

9. Nucifora G, Aquaro GD, Pingitore A, Masci PG, Lombardi M. Myocardial fibrosis in isolated left ventricular non-compaction and its relation to disease severity. Eur J Heart Fail. 2011;13(2):170-6. doi: 10.1093/eurjhf/hfq222. [PubMed: 21208941].

10. Weiford BC, Subbarao VD, Mulhern KM. Noncompaction of the ventricular myocardium. Circulation. 2004;109(24):2965-71. doi: 10.1161/01.CIR.0000132478.60674.Do. [PubMed:15210614].

11. Agarwal A, Khandheria BK, Paterick TE, Treiber SC, Bush M, Tajik AJ. Left ventricular noncompaction in patients with bicuspid aortic valve. J Am Soc Echocardiogr. 2013;26(11):1306-13. doi: 10.1016/j.echo.2013.08.003. [PubMed: 24044978].

12. Ali SK, Abu-Sulaiman R, Agouba RB. Noncompaction cardiomyopathy: A new mechanism for mitral regurgitation with distinct clinical, echocardiographic features and pathological correlations. J Saudi Heart Assoc. 2015;27(2):71-8. doi: 10.1016/j.jsha.2014.07.002. [PubMed: 25870499]. [PubMed Central: PMC4392355]. 